\title{
季鏻和金属对高张力六元环的双重稳定化作用
}

\author{
朱从青＼cjkstart曹晓宇* 夏海平* \\ (厦门大学化学化工学院 厦门 361005)
}

\begin{abstract}
摘要 环炔和环内累积多烯作为有机合成的重要中间体，在合成化学中扮演着重要的角色. 但是，对这类高活性物种 的稳定化一直是学术界研究的热点和难点. 近年, 我们课题组发现季鏻和过渡金属具有双重稳定化作用, 可以极好地 稳定这类高张力环状化合物. 对我们课题组在这方面的最新进展进行了综述.
\end{abstract}

关键词 芳炔; 金属杂环; 季鏻; 稳定化; 张力环

\section{Double Stabilization of Highly Strained Six-Membered Rings by Phosphonium and Transition Metal}

\author{
Zhu, Congqing Cao, Xiaoyu* Xia, Haiping* \\ (College of Chemistry and Chemical Engineering, Xiamen University, Xiamen 361005)
}

\begin{abstract}
Cycloalkynes and cyclocumulenes have attracted considerable attention because they are key intermediates in organic synthesis. Stabilization of these highly active species remains a challenge for synthetic chemists. Recently, we found that phosphonium and transition metal replacement can double stabilize these species. Using this strategy, a series of highly strained six-membered rings with remarkable stability have been synthesized. Herein, the latest progress of our group is reviewed.
\end{abstract}

Keywords arynes; metallacycles; phosphonium; stabilization; strained rings

高活性物种(如: 环内累积多烯、芳炔等)是有机反应 的重要中间体 ${ }^{[1 \sim 4]}$, 对它们的分离与表征一直是合成化学 研究的热点与难点. 作为苯的同分异构体, 1,2,4-环已三 烯又称为 “异苯” (Chart 1), 因其含有环内累积双键而极 不稳定 ${ }^{[4]}$. 苯炔和吡啶炔(Chart 1)作为两类最古老、研究 最透彻的芳炔化合物 ${ }^{[1]}$, 是众多药物合成的重要前体 ${ }^{[5]}$. 但是，由于六元环内碳碳参键所导致的环张力使得这类 芳炔化合物极不稳定, 至今仍未能被分离得到.
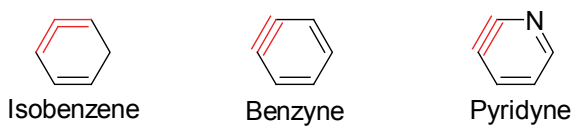

Chart 1

金属的引入可以降低环张力, 是稳定环炔的一种有 效方法 ${ }^{[6]}$. 例如: 2001 年, Jia 课题组 ${ }^{[6]}$ 通过引入一个金 属骨架，成功地分离到首例金属苯炔化合物. 2002 年,
Suzuki 课题组 ${ }^{[66]}$ 报道了金属杂五元环炔的合成. 2004 年, Esteruelas 课题组 ${ }^{[7]}$ 报道了首例金属杂异苯的合成. 近年来, 我们发现在引入金属的同时, 在主环上引 入季鏻取代基，可以进一步提高化合物的稳定性. 采用 这种方法，不仅可以合成具有高度热稳定性的金属杂 苯 ${ }^{[8]}$, 还可以稳定经典有机中多种重要的活性中间体, 如异苯 ${ }^{[9]}$ 、苯炔 ${ }^{[10]}$ 和吡啶炔 ${ }^{[11]}$. 这些金属杂环化合物在 空气氛中均具有较高的热稳定性. 本文将综述本课题组 在这方面的最新进展, 并展望今后的研究方向.

\section{1 异金属苯}

苯是最稳定的芳香化合物. 而它的同分异构体一 1,2,4-环已三烯(简称 “异苯”) 键长趋于定域, 已经不具 备芳香性. 且因存在环内累积双键, 张力较大, 因此很 不稳定, 只是作为反应的中间体存在. 2004 年, Esteruelas 等 ${ }^{[7]}$ 合成了金属中心 16 电子的金属杂异苯, 简称“异

\footnotetext{
*E-mail: xcao@xmu.edu.cn,hpxia@xmu.edu.cn

Received January 31, 2013; revised March 21, 2013; published online March 22, 2013.

Project supported by the National Basic Research Program of China (No. 2012CB821600), the National Natural Science Foundation of China (Nos. 21174115, 20925208) and the Program for Changjiang Scholars and Innovative Research Team in University.

国家重点基础研究发展计划(No. 2012CB821600)、国家自然科学基金(Nos. 21174115, 20925208)及长江学者和创新团队发展计划资助项目.
} 
金属苯” (Chart 2).

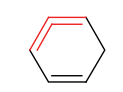

Isobenzene

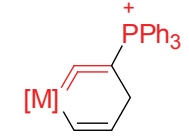

Isometallabenzene

Chart 2

最近我们发现, 在引入金属的同时在异苯环上引入 季鏻取代基，可以构筑稳定的异金属苯 ${ }^{[9]}$. 由含金属氢 的锇亚乙烯化合物 $\mathbf{1}$ 与末端炔醇 $\mathrm{HC} \equiv \mathrm{CCH}(\mathrm{OH}) \mathrm{Ph}$ 发生 形式上的 $[3+3]$ 关环反应, 可以较高的收率得到稳定的 异金属苯 2 (Eq. 1), 其金属中心满足 18 电子规则.

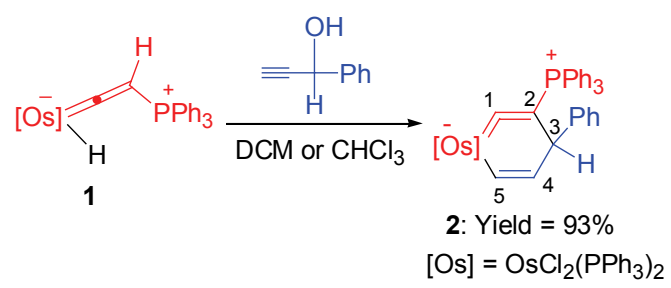

单晶结构表明, $\mathrm{Os}-\mathrm{C}(1)$ 键长为 $1.781 \AA$, 位于典型 的 $\mathrm{Os}-\mathrm{C}$ 双键键长 $(1.78 \sim 2.14 \AA)$ 范围内 ${ }^{[12]}$. $\mathrm{C}(1)-$ $C(2)$ 和 $C(4)-C(5)$ 键长分别为 1.339 和 $1.351 \AA$, 是典型 的 $\mathrm{C}-\mathrm{C}$ 双键. 而 $\mathrm{C}(2)-\mathrm{C}(3)$ 和 $\mathrm{C}(3)-\mathrm{C}(4)$ 键长分别为 1.563 和 $1.507 \AA$, 是典型的 $\mathrm{C}-\mathrm{C}$ 单键. 因此, 化合物 2 是明显的定域结构. 虽然 2 中含有一个 $\mathrm{sp}^{3}$ 杂化碳原子, 但其具有很好的共平面性, C(3)原子偏离拟合平面的偏 差最大, 为 $0.0571 \AA$.

异金属苯 2 具有较好的热稳定性, 其固体样品在空 气氛中加热到 $100{ }^{\circ} \mathrm{C}$ 可以维持 $5 \mathrm{~h}$ 不分解. 化合物 2 在 溶液状态下也可以稳定存在, 只有在回流条件下, 2 才 可以向相应的 $\mathrm{Cp}$ 配合物转变(Scheme 1).

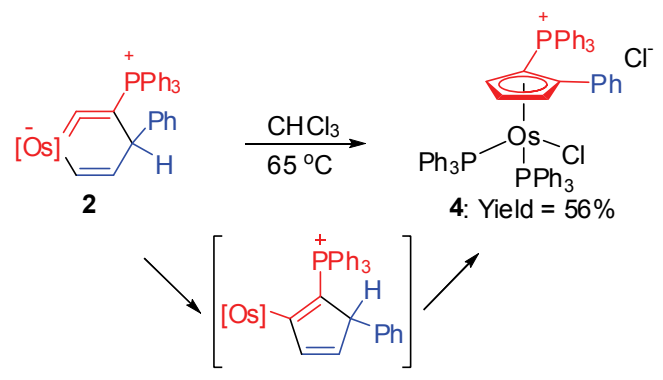

3

$[\mathrm{Os}]=\mathrm{OsCl}_{2}\left(\mathrm{PPh}_{3}\right)_{2}$

Scheme 1

在异金属苯 2 中, 金属所在位置的夹角 [C(1)$\mathrm{Os}-\mathrm{C}(2)$ ] 只有 $78.3^{\circ}$, 远小于六边形的内角 $\left(120^{\circ}\right)$. 而 $\mathrm{Os}-\mathrm{C}(1)-\mathrm{C}(2)$ 的夹角为 $155.1^{\circ}$, 与 Esteruelas 等 ${ }^{[7]}$ 报道
的异金属苯相同位置的夹角相当. 因此, 金属的引入, 使环内累积双键的弯曲程度减小. 同时，季鏻的参与， 产生了如 Eq. 2 所示的共振结构式, 进一步缓解了环内 累积双键导致的张力, 从而使 2 具有较好的热稳定性.

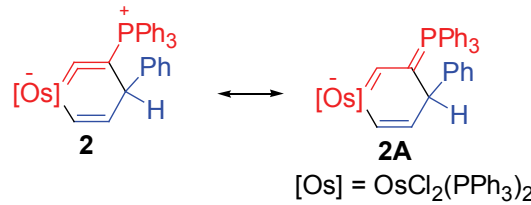

为了进一步研究异金属苯 $\mathbf{2}$ 稳定性的来源，对其简 化模型 $2^{\prime}$ (用 $\mathrm{PH}_{3}$ 替代 $\mathrm{PPh}_{3}$ )进行了理论分析. 首先, 研 究了化合物 $\mathbf{2}^{\prime}$ 与首例异金属苯 $5^{\prime}$ 稳定性的差别. 如 Chart 3 所示, 选取相应的 $\mathrm{Cp}$ 配合物 (4' $\mathbf{4}^{\prime}$ 和 $\left.\mathbf{6}^{\prime}\right)$ 作为能量零 点, $\mathbf{2}^{\prime}$ 和 $\mathbf{5}^{\prime}$ 的相对能量分别为 49.0 和 $159.8 \mathrm{~kJ} \cdot \mathrm{mol}^{-1}$. 因 此，异金属苯 2 具有更高的热稳定性.<smiles>PC1C=CC([PH3+])C(P)(c2ccccc2)C1P</smiles>

2': 49.0

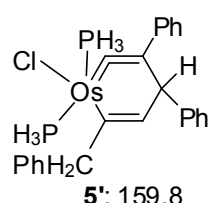

5': 159

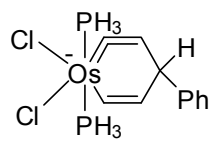

2'- $\mathrm{PH}_{3}^{+}: 110.0$

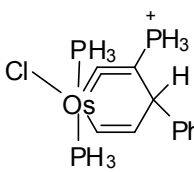

2'-Cl: 144.3

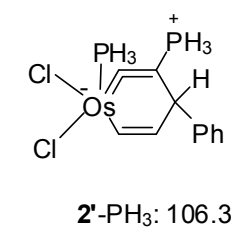

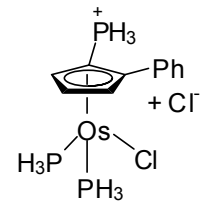

$4^{\prime}: 0.0$

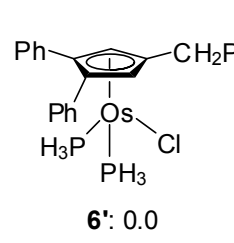

6': 0.0<smiles>Oc1ccccc1-c1ccccc1</smiles>

$4^{\prime}-\mathrm{PH}_{3}{ }^{+}: 0.0$<smiles>FP(F)(P)(Cl)c1cc(P)cc(P)c1</smiles><smiles>[Pb]c1cccc([Pb])c1</smiles>

\section{Chart 3}

与首例异金属苯 $\mathbf{5}$ 相比，化合物 $\mathbf{2}$ 中主环上庞大的 季鏻取代基和金属中心 18 电子结构可能是其稳定的主 要原因. 如 Chart 3 所示：主环结构上不含季鏻取代基的 异金属苯 $2^{\prime}-\mathrm{PH}_{3}{ }^{+}$比相应 $\mathrm{Cp}$ 配合物 $4^{\prime}-\mathrm{PH}_{3}{ }^{+}$的自由能高 $110.0 \mathrm{~kJ} \bullet \mathrm{mol}^{-1}$. 两种金属中心 16 电子的异金属苯 $2^{\prime}-\mathrm{Cl}^{-}$ 和 $2^{\prime}-\mathrm{PH}_{3}$ 比相应 $\mathrm{Cp}$ 配合物 $4^{\prime}-\mathrm{Cl}^{-}$和 $4^{\prime}-\mathrm{PH}_{3}$ 的自由能分 别高 144.3 和 $106.3 \mathrm{~kJ} \cdot \mathrm{mol}^{-1}$. 因此，季鏻取代基和金属 中心 18 电子结构对产物稳定性有较大的影响.

总之，我们通过含金属氢的锇亚乙烯化合物与末端 炔醇发生形式上的 $[3+3]$ 关环反应，合成了稳定的异金 属苯. 理论计算表明, 季鏻取代基和金属中心 18 电子结 构是产物具有高稳定性的主要因素. 


\section{2 金属苯炔}

苯炔是有机化学中一类重要中间体, 可用于众多功 能有机化合物的合成 ${ }^{[2,13]}$. 与苯和异苯相比, 苯炔增加 了一个不饱和度. 同时, 因其含有六元环内碳碳参键而 极不稳定. 苯炔目前还未能被分离出来, 只能从光谱上 得到表征. 过渡金属因其具有较大的原子半径, 可用于 缓解环内参键导致的环张力. 通过在苯炔环内引入过渡 金属这种方法, 2001 年 Jia 课题组 ${ }^{[6]}$ 合成了首例金属苯 炔化合物(Chart 4). 首例金属苯炔的成功分离改变了人 们对此类化合物稳定性差的传统观点. 理论计算表明, 过渡金属的引入以及大取代基的存在都是其稳定的因 素 ${ }^{[14]}$. 随后，他们又合成了一系列锇苯炔、锞苯炔化合 物 $^{[15]}$.

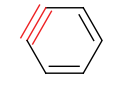

Benzyne

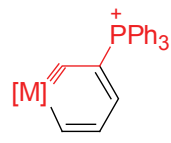

Metallabenzyne

\section{Chart 4}

在尝试合成含有不同取代基的异金属苯时，我们发 现: 如果异金属苯 I 中含有较好的离去基团(Scheme 2), 则可以生成含有较高不饱和度的金属杂六元环化合物 II, 它是金属苯炔 III 的一种共振结构式. 这可以认为是 合成金属苯炔的一种新方法 ${ }^{[10]}$.

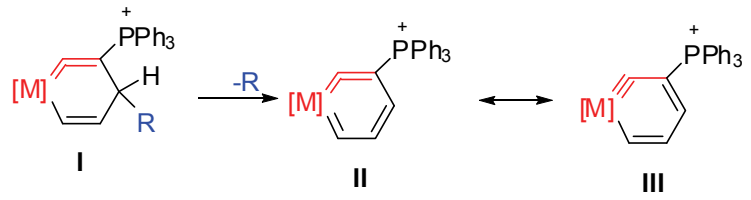

Scheme 2

用含金属氢的锇亚乙烯化合物 $\mathbf{1}$ 与含有双易离去基 团的炔烃 $\mathrm{HC} \equiv \mathrm{CCH}(\mathrm{OEt})_{2}$ 反应，可以一锅法合成金属 苯炔化合物 8 (Scheme 3). 通过核磁和高分辨质谱监测 发现, 8 的生成经历了异金属苯中间体 7.

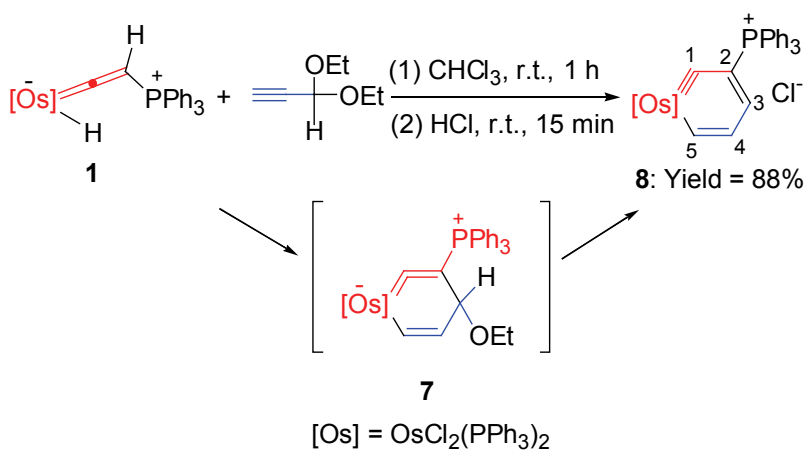

Scheme 3
化合物 8 的金属杂六元环具有较好的共平面性. $\mathrm{Os}-\mathrm{C}(1)-\mathrm{C}(2)$ 夹角为 $148.6^{\circ}, \mathrm{Os}-\mathrm{C}(1)$ 键长为 $1.775 \AA$, 环内 $\mathrm{C}-\mathrm{C}$ 键长位于 $1.381 \sim 1.403 \AA$ 范围内, 介于碳碳 单键和双键之间, 无明显单双键交替, 意味着其具有很 好的离域结构. 化合物 $\mathbf{8}$ 中键长、键角数据与已报道的 锇苯炔中键长、键角数据相一致 ${ }^{[6 a, 15]}$.

化合物 8 分子内含有环内金属碳参键，但化合物 8 仍然具有较好的热稳定性, 其固体样品在空气氛中、 120 ${ }^{\circ} \mathrm{C}$ 下加热 $5 \mathrm{~h}$ 不会发生改变. 即使在溶液状态下，也可 以在 $80{ }^{\circ} \mathrm{C}$ 加热 $8 \mathrm{~h}$ 而没有明显变化. 这种良好的热稳 定性不仅是因为金属的引入，极大地缓解了六元环内参 键导致的环张力. 同时, $\mathrm{C}(2)$ 原子上季鏻取代基的立体 效应和电子效应也发挥着至关重要的作用. 类似的稳定 化效应在异金属苯的稳定性中已经通过理论计算得以 证实 ${ }^{[9 a]}$.

化合物 8 与常见的酸 $\left(\mathrm{HOAc}, \mathrm{H}_{3} \mathrm{PO}_{4}, \mathrm{HBF}_{4}\right.$ 和 $\mathrm{HCl}$ )、 碱 $\left(\mathrm{K}_{2} \mathrm{CO}_{3}\right.$ 和 $\left.\mathrm{NaOH}\right) 、$ 亲核试剂 $\left(\mathrm{H}_{2} \mathrm{O}, \mathrm{MeOH}\right.$ 和 $\left.\mathrm{NaBH}_{4}\right)$ 都不反应. 但是, 亲核能力较强的 $\mathrm{EtLi}$ 可以与 $\mathbf{8}$ 反应, 生成异金属苯 9 (Eq. 3). 在这个反应中, $\mathrm{Et}^{-}$选择性地进 攻金属苯炔 8 的 C(3)位置.
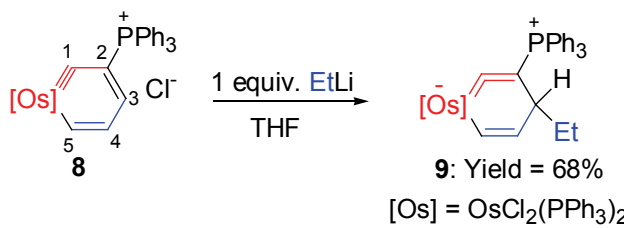

之前的理论计算表明, 金属苯炔的 $\mathrm{C}(1), \mathrm{C}(3)$ 和 $C(5)$ 位置均可以受亲核试剂进攻 ${ }^{[14]}$. 其中 $C(1)$ 位置具有 较大的张力, 最容易与亲核试剂反应, 这与 Jia 课题 组 ${ }^{[16]}$ 报道的亲核试剂选择性进攻金属苯炔的卡拜碳原 子 $[\mathrm{C}(1)]$ 相符. 但是, 在金属苯炔 8 中, 由于 $\mathrm{C}(1)$ 位置被 三个庞大的 $\mathrm{PPh}_{3}$ 所包裹，阻止了其与亲核试剂的反应. 同时，通过理论计算发现， C(3)位置对化合物 8 的最低 未占据轨道的贡献最大. 因此, 亲核试剂会优先进攻化 合物 8 的 C(3)位置.

因此，我们通过季鏻取代基和过渡金属的双重稳定 化作用，合成了稳定的六元环炔一一金属苯炔化合物， 并且发现其具有不同于之前所报道的金属苯炔的反应性.

\section{3 金属吡啶炔}

苯炔的含氮类似物——吡啶炔, 也是有机合成的重 要中间体, 特别是用于合成多取代的吡啶衍生物 ${ }^{[3,17]}$. 与苯炔类似，吡啶炔也是非常活泼的中间体，从未被成 功分离. 虽然目前有多例金属苯炔化合物的合成报道, 但是, 金属吡啶炔一直未见报道. 我们课题组 ${ }^{[11]}$ 发现在 
引入金属的同时, 在主环上引入季鏻取代基, 可以有效 地稳定吡啶炔化合物(Chart 5).

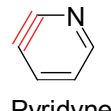

Pyridyne

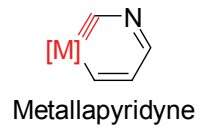

Chart 5

如 Scheme 4 所示: 含 SCN 取代基的锇苯配合物 $\mathbf{1 0}$ 在四氢呋喃中与过量的苯乙炔基锂反应, 得到锇苯衍生 物 11. 在碘的促进下, 11 金属中心的 SCN 配体参与环化 得到金属杂吡啶衍生物 12.12 在还原剂 $\mathrm{CuCl}$ 的作用下, 脱除与金属中心相连的硫原子, 得到首例含有金属吡啶 炔结构的衍生物 13. 与异金属苯 $\mathbf{2}$ 、金属苯炔 $\mathbf{8}$ 不同的是, 在金属吡啶炔衍生物 13 中, 季鏻取代基位于金属吡啶炔 相邻的六元环上，增加了并六元环化合物的稳定性.

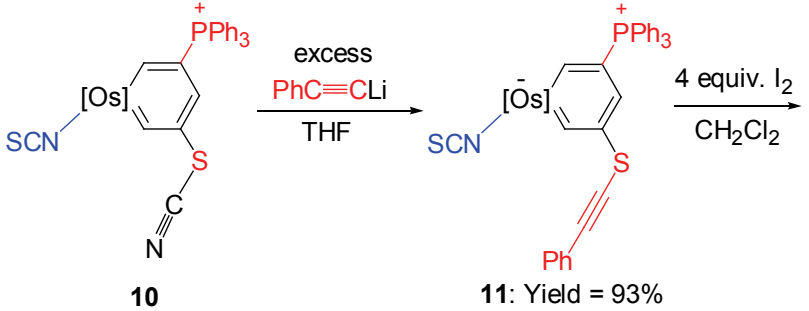

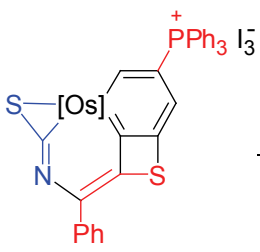

12: Yield $=65 \%$

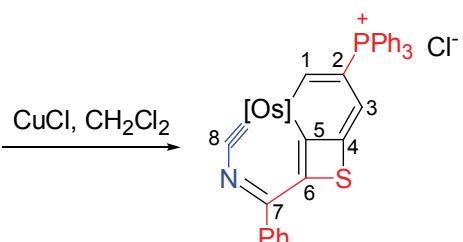

13: Yield $=80 \%$
[Os] $=\mathrm{Os}(\mathrm{SCN})\left(\mathrm{PPh}_{3}\right)_{2}$

\section{Scheme 4}

有趣的是，在化合物 12 和 13 中，氮原子均位于金 属的间位(Chart 6, B). 而之前所报道的两例金属吡啶化 合物的氮原子均位于金属的邻位 $(\text { Chart } 6, \mathbf{A})^{[18]}$. 因此, 化合物 12 和 13 可以认为是一类新颖的金属吡啶和金属 吡啶炔衍生物.

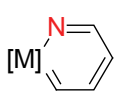

A

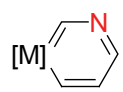

B

\section{Chart 6}

金属吡啶炔 13 的单晶结构显示两个并六元环具有 较好的共平面性. 其中 $\mathrm{Os}-\mathrm{C}(8)$ (1.894 $\AA$ ) 比已报道的 $\mathrm{Os}-\mathrm{C}$ 参键键长 $(1.671 \sim 1.815 \AA)$ 更长, 这可能是因为六 元环的张力导致了 $\mathrm{Os}-\mathrm{C}$ 参键的拉伸. 结合环内其它 $\mathrm{Os}-\mathrm{C}$ 和 $\mathrm{C}-\mathrm{C}$ 键长, 我们推测化合物 13 具有如 $\mathrm{Eq} .4$
所示的两种主要共振结构式.

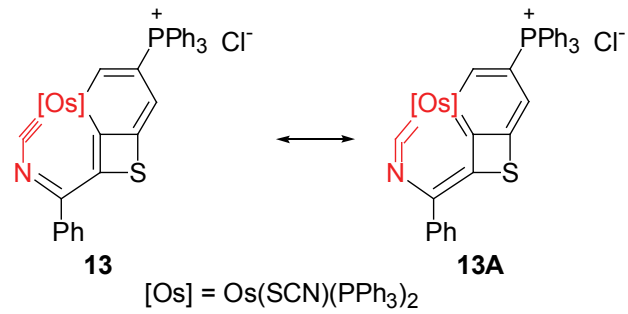

化合物 12 和 13 具有很好的热稳定性, 其溶液可以 在空气氛中放置数月而不发生分解，固体样品可以在空 气氛中 $100{ }^{\circ} \mathrm{C}$ 加热 $5 \mathrm{~h}$ 几乎不发生变化. 虽然化合物 $\mathbf{1 3}$ 中含有金属碳参键, 但是它和常见的亲核试剂 $\left(\mathrm{H}_{2} \mathrm{O}\right.$, $\mathrm{MeOH}, \mathrm{MeONa}, \mathrm{KOH}$ 和 $\mathrm{NaSH}$ )及亲电试剂 $\left(\mathrm{HCl}, \mathrm{HBF}_{4}\right.$, $\mathrm{O}_{2}, \mathrm{MeI}$ 和 MeOTf)都不发生反应.

理论计算评估了化合物 13 的芳香性. 首先, 13 的芳 香稳定化能为 $47.3 \mathrm{~kJ} / \mathrm{mol}$, 位于已报道的金属杂芳香化 合物芳香稳定化能 $\left(157.3 \sim 28.5 \mathrm{~kJ} \cdot \mathrm{mol}^{-1}\right)$ 的低端 ${ }^{[19]}$. 同 时, 金属吡啶炔所在六元环的核独立化学位移 ${ }^{[20]}$ (NICS)值为 $\delta-4.5$, 与已报道的金属苯的 NICS 值相 近 ${ }^{[21]}$. 因此, 结合化合物 $\mathbf{1 3}$ 的核磁数据、单晶键长等实 验数据, 推测其具有一定的芳香性.

因此，我们通过季鏻取代基和过渡金属的引入，稳 定了经典有机化学中重要的吡啶炔中间体. 金属吡啶炔 的芳香性进一步增加了它的稳定性.

\section{4 总结与展望}

随着季鏻取代基和过渡金属的引入，我们课题组成 功地稳定了多类经典有机化学中的活性中间体，如异 苯、苯炔和吡啶炔. 在这些体系中，过渡金属的引入可 以降低环内参键或累积双键导致的环张力，同时，庞大 的季鏻取代基又有着很好的空间保护效应. 为经典有机 中高活性中间体的捕捉提供了一种有效的策略.

异苯、苯炔和吡啶炔都是经典有机中可以通过光谱 或化学反应证实的中间体. 但是, 在有机体系中还存在 一些目前尚未能得到较好证实的高活性中间体(Chart 7). 借助季鏻取代基和过渡金属的双重稳定化作用，可 能会稳定更多经典有机化学中未曾分离出来的活性中 间体. 同时, 通过这种双重稳定化策略，一些经典有机 中未曾观测到的中间体(如小环炔和环内累积多烯)也将 有可能被分离得到.

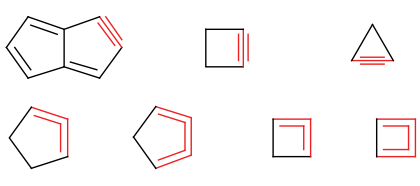

Chart 7 
季鏻取代基和过渡金属的引入，大大增加了此类高 张力环状化合物对空气、水、热的稳定性. 同时, 这些 稳定的金属有机化合物合成方便. 鉴于这些特点, 我们 未来将考察这类金属杂共轭体系在生物成像、分子探 针、太阳能电池等领域的应用前景.

\section{作者简介}

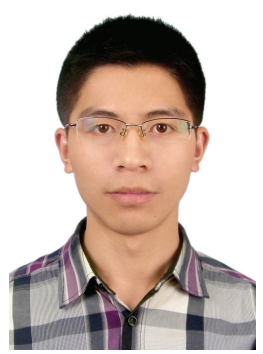

朱从青, 在读博士研究生. 1986 年出生于安徽省安 庆市. 2008 年安庆师范学院本科毕业, 同年进入厦门大 学夏海平教授课题组攻读博士学位. 研究方向为金属杂 芳环化合物的合成、表征及反应性研究. 目前已合成各 类金属杂环化合物 60 余例, 并于 2012 年 10 月在全国金 属有机化学学术讨论会上做了题为 “金属桥位稠环芳香 化合物的合成” 的口头报告.

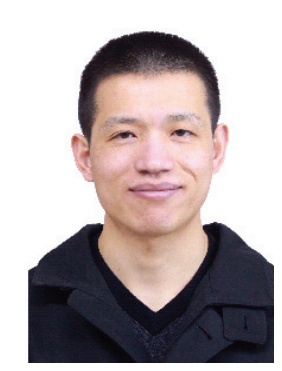

曹晓宇, 博士, 副教授. 1981 年出生于福建省龙岩 市. 2003 年北京大学化学与分子工程学院本科毕业(导 师: 裴坚教授). 2009 年法国斯特拉斯堡大学超分子科学 与工程研究所博士毕业(导师: Jean-Marie Lehn 教授). 2008 年底获玛丽居里奖学金资助, 在荷兰埃因霍温理 工大学生物医学与工程系从事博士后研究(合作导师: E. W. Meijer 教授). 2011 获聘厦门大学化学化工学院副教 授. 主要从事新型芳香化合物的设计、合成、自组装及 其光电磁材料应用方面的研究.

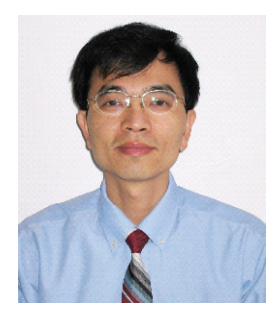

夏海平，博士，教授. 1964 年出生于上海市. 1983 年
厦门大学化学系本科毕业, 1986 年厦门大学化学系硕士 毕业, 之后留校任教. 1995 1997 年在香港科技大学化 学系从事访问研究. 1999 年晋升为教授. 2002 年获得厦 门大学理学博士学位. 2004 年入选教育部首批 “新世纪 优秀人才支持计划”. 2009 年获国家杰出青年科学基金. 2010 年获聘福建省 “闽江特聘教授” . 主要从事金属有 机共轭体系的合成方法学研究. 开拓了一系列构筑金属 杂环的新方法—- $5+1],[4+2]$ 和 $[3+3]$ 关环法等. 已 正式发表学术论文百余篇, 通讯作者论文曾被美国化学 会的 “C\&E News”, “Heart Cut” , “Noteworthy Chemistry” 和英国皇家化学会的 “Chemistry World” 等 报道. 欢迎访问课题组主页：http://chem.xmu.edu.cn/ group/hpxia/index.htm.

\section{References}

[1] Wenk, H. H.; Winkler, M.; Sander, W. Angew. Chem., Int. Ed. 2003, 42, 502 .

[2] Hoye, T. R.; Baire, B.; Niu, D.; Willoughby, P. H.; Woods, B. P. Nature 2012, 490, 208.

[3] Goetz, A. E.; Garg, N. K. Nat. Chem. 2013, 5, 54.

[4] (a) Jaz, A.; Bradley, A. Z.; Burrell, R. C.; Li, W. H. H.; Daoust, K. J.; Bovee, L. B.; DiRico, K. J.; Johnson, R. P. J. Org. Chem. 2011, 76,9320 .

(b) Engels, B.; Schöneboom, J. C.; Münster, A. F.; Groetsch, S.; Christl, M. J. Am. Chem. Soc. 2002, 124, 287.

(c) Burrell, R. C.; Daoust, K. J.; Bradley, A. Z.; DiRico, K. J.; Johnson, R. P. J. Am. Chem. Soc. 1996, 118, 4218.

[5] (a) Smith, A. B. III; Kim, W.-S. Proc. Natl. Acad. Sci. U. S. A 2011, 108, 6787.

(b) Allan, K. M.; Stoltz, B. M. J. Am. Chem. Soc. 2008, 130, 17270. (c) Carroll, F. I.; Robinson, T. P.; Brieaddy, L. E.; Atkinson, R. N.; Mascarella, S. W.; Damaj, M. I.; Martin, B. R.; Navarro, H. A. J. Med. Chem. 2007, 50, 6383.

[6] (a) Wen, T. B.; Zhou, Z. Y.; Jia, G. Angew. Chem., Int. Ed. 2001, 40, 1951.

(b) Suzuki, N.; Nishiura, M.; Wakatsuki, Y. Science 2002, 295, 660 .

(c) Rosenthal, U. Angew. Chem., Int. Ed. 2004, 43, 3882.

(d) Jia, G. Coord. Chem. Rev. 2007, 251, 2167.

(e) Suzuki, N.; Hashizume, D. Coord. Chem. Rev. 2010, 254, 1307.

[7] Barrio, P.; Esteruelas, M. A.; Oñate, E. J. Am. Chem. Soc. 2004, $126,1946$.

[8] (a) Xia, H.; He, G.; Zhang, H.; Wen, T. B.; Sung, H. H. Y.; Williams, I. D.; Jia, G. J. Am. Chem. Soc. 2004, 126, 6862.

(b) Zhang, H.; Xia, H.; He, G.; Wen, T. B.; Gong, L.; Jia, G. Angew. Chem., Int. Ed. 2006, 45, 2920.

(c) Lin, R.; Zhang, H.; Li, S.; Wang, J.; Xia, H. Chem.-Eur. J. 2011, 17, 4223 .

[9] Zhao, Q.; Gong, L.; Xu, C.; Zhu, J.; He, X.; Xia, H. Angew. Chem., Int. Ed. 2011, 50, 1354.

[10] Zhao, Q.; Zhu, J.; Huang, Z.-A.; Cao, X.-Y.; Xia, H. Chem.-Eur. J. 2012, 18, 11597.

[11] Wang, T.; Zhang, H.; Han, F.; Lin, R.; Lin, Z.; Xia, H. Angew. Chem., Int. Ed. 2012, 51, 9838.

[12] (a) Based on a search of the Cambridge Structural Database, 2011, CSD version 5.33. 
(b) Bolaño, T.; Castarlenas, R.; Esteruelas, M. A.; Oñate, E. Organometallics 2007, 26, 2037.

(c) Castarlenas, R.; Esteruelas, M. A.; Oñate, E. Organometallics 2007, 26, 2129.

[13] (a) Wenk, H. H.; Winkler, M.; Sander, W. Angew. Chem., Int. Ed. 2003, 42, 502.

(b) Peña, D.; Pérez, D.; Guitián, E. Angew. Chem., Int. Ed. 2006, 45,3579 .

(c) Bhunia, A.; Yetra, S. R.; Biju, A. T. Chem. Soc. Rev. 2012, 41, 3140 .

(d) Bhojgude, S. S.; Biju, A. T. Angew. Chem., Int. Ed. 2012, 51, 1520 .

[14] (a) Ng, S. M.; Huang, X.; Wen, T. B.; Jia, G.; Lin, Z. Organometallics 2003, 22, 3898.

(b) Yang, S. Y.; Li, X. Y.; Huang, Y. Z. J. Organomet. Chem. 2002, 658,9 .

[15] (a) Chen, J.; Sung, H. H. Y.; Williams, I. D.; Lin, Z.; Jia, G. Angew. Chem., Int. Ed. 2011, 50, 10675.

(b) Chen, J.; Shi, C.; Sung, H. H. Y.; Williams, I. D.; Lin, Z.; Jia, G. Angew. Chem., Int. Ed. 2011, 50, 7295.

(c) He, G.; Zhu, J.; Hung, W. Y.; Wen, T. B.; Sung, H. H. Y.; Williams, I. D.; Lin, Z.; Jia, G. Angew. Chem., Int. Ed. 2007, 46, 9065.

(d) Wen, T. B.; Hung, W. Y.; Sung, H. H. Y.; Williams, I. D.; Jia, G. J. Am. Chem. Soc. 2005, 127, 2856.

(e) Wen, T. B.; Ng, S. M.; Hung, W. Y.; Zhou, Z. Y.; Lo, M. F.; Shek, L. Y.; Williams, I. D.; Lin, Z.; Jia, G. J. Am. Chem. Soc.
$2003,125,884$.

[16] Hung, W. Y.; Zhu, J.; Wen, T. B.; Yu, K. P.; Sung, H. H. Y.; Williams, I. D.; Lin, Z.; Jia, G. J. Am. Chem. Soc. 2006, 128, 13742.

[17] (a) Goetz, A. E.; Bronner, S. M.; Cisneros, J. D.; Melamed, J. M.; Paton, R. S.; Houk, K. N.; Garg, N. K. Angew. Chem., Int. Ed. 2012, 51, 2758.

(b) Jiang, L.; Yu, X.; Fang, B.; Wu, J. Org. Biomol. Chem. 2012, 10, 8102 .

(c) Fang, Y.; Larock, R. C. Tetrahedron 2012, 68, 2819.

(d) Winkler, M.; Cakir, B.; Sander, W. J. Am. Chem. Soc. 2004, 126, 6135 .

[18] (a) Weller, K. J.; Filippov, I.; Briggs, P. M.; Wigley, D. E. Organometallics 1998, 17, 322.

(b) Liu, B.; Wang, H.; Xie, H.; Zeng, B.; Chen, J.; Tao, J.; Wen, T.; Cao, Z.; Xia, H. Angew. Chem., Int. Ed. 2009, 48, 5430.

[19] (a) De Proft, F.; Geerlings, P. Phys. Chem. Chem. Phys. 2004, 6, 242.

(b) Mauksch, M.; Tsogoeva, S. B. Chem.-Eur. J. 2010, 16, 7843.

[20] (a) Jiao, H.; Schleyer, P. v. R.; Mo, Y.; McAllister, M. A.; Tidwell, T. T. J. Am. Chem. Soc. 1997, 119, 7075.

(b) Schleyer, P. V. R.; Maerker, C.; Dransfeld, A.; Jiao, H.; Hommes, N. J. R. V. E. J. Am. Chem. Soc. 1996, 118, 6317.

(c) Chen, Z.; Wannere, C. S.; Corminboeuf, C.; Puchta, R.; Schleyer, P. V. R. Chem. Rev. 2005, 105, 3842.

[21] Iron, M. A.; Lucassen, A. C. B.; Cohen, H.; van der Boom, M. E.; Martin, J. M. L. J. Am. Chem. Soc. 2004, 126, 11699.

(Zhao, C.) 